\title{
A Prospective Study of Renal Calculi and Its Metabolic Evaluation
}

\author{
T. Rudra Prasad Reddy ${ }^{1}, \mathrm{R}$ V Bhosle ${ }^{1}$ \\ ${ }^{1}$ Associate Professor, Department of General Surgery, MNR Medical College and Hospital, Sangareddy, Telangana, India \\ Corresponding author: R V Bhosle, Associate Professor, Department of General Surgery, MNR Medical College and Hospital, \\ Sangareddy, India
}

DOI: $10.21276 /$ ijcmsr.2018.3.3.31

How to cite this article: T. Rudra Prasad Reddy, R V Bhosle. A prospective study of renal calculi and its metabolic evaluation. International Journal of Contemporary Medicine Surgery and Radiology. 2018;3(3):C143-C145.

\section{A B S T R A C T}

Introduction: Kidney stones develop as a result of various metabolic disorders which affect various elements in the body especially calcium. Study was done to record the metabolic evaluation of kidney stone patients and to identify metabolic abnormality, and treating the same.

Material and Methods: A prospective observational study, of hundred patients with the history of pain in flanks radiating to front and analyzing them for any metabolic abnormality.

Results: Out of the 100 patients, 70 were males and 30 were females. Patients underwent metabolic evaluation, in which 55 male, 15 female patients had a metabolic abnormality remaining male and female patients had no abnormality. The common abnormality found was hyperuricosuria, followed by hypercalciuria.

Conclusion: Metabolic evaluation is a must which greatly help to diagnose kidney stone and reduce the risk of stone recurrence

Keywords: Kidney Stones, Metabolic evaluation, Hyperuricosuria, Hypercalciuria

\section{INTRODUCTION}

Kidney stones are made up of mainly uric acid, calcium oxalate, triple phosphate or cystine. They are solid in structures, based on their composition four kinds of stones mainly- 1. calcium oxalate stones 2 . uric acid stones 3 . cystine stones and 4 . struvite stones. ${ }^{1}$ Renal calculi are more common in males than in females. Mostly in 30-60yrs age group are affected. A genetic defect in urinary acidification associated with a high frequency of stone formation. ${ }^{2}$ family history of stone disease, individuals who drink less water than the recommended, recurrent urinary tract infections (UTIs), renal tubular acidosis (RTA) and hyperparathyroidism, People with certain medical conditions, such as hypertension, diabetes, gout, and those who take certain medications, are the major risk factors. Symptoms include pain in the back radiating to front, blood in urine, vomiting and nausea, burning urination, urgency, fever and chills. ${ }^{3}$ Among kidney stones Calcium oxalate and calcium phosphate are the most common types accounting for $>70-80 \%$ of stones, followed by uric acid (8-10\%) and cysteine, struvite. Renal calculi both medical and surgical treatment. In $98 \%$ of cases stones passes spontaneously of diameter $5 \mathrm{~mm}$, while those stones measuring 6 to $10 \mathrm{~mm}$ in diameter pass spontaneously in less than $53 \%$ of cases. ${ }^{4}$ Large size stones require surgical intervention. Metabolic evaluation (ME) of renal stones, have become an important part as it helps to identify the major metabolic abnormalities. Metabolic Abnormality when treated properly can reduce the formation and recurrence of stones. Metabolic screening is based on diet related and helps in stone identification, points out stone risk factors, and identifies patient's comorbidity. $\mathrm{ME}$ includes tests for serum calcium, phosphorus, uric acid, creatinine, blood urea nitrogen (BUN), electrolytes, CUE and urine $\mathrm{pH}$. 24-hrs urine collections on a random diet an essential part of metabolic evaluation. ${ }^{5}$ It includes various components of urine such as urine volume, calcium, sodium, creatinine, citrate, uric acid, and oxalate. Study was done to record the metabolic evaluation of kidney stone patients and to identify metabolic abnormality, and treating the same.

\section{MATERIAL AND METHODS}

From June 2017 to November 2017, a prospective observational study was done, out of 100 patients with the history of pain in flanks radiating to front. 70 patients with metabolic abnormality, were included in our study remaining patients with no abnormality were excluded. The study was conducted over a time of 6 months, starting from June 2017 to November 2017 in the MNR Medical College And Hospital, Sangareddy, after ethical approval has been obtained from the human ethics committee and Informed consent was obtained from all individual participants were included in the study.

This was a hospital-based prospective observational study, enrolling 70 patients presenting with kidney stones, to the department of General Surgery of the hospital. age, sex, 


\begin{tabular}{|l|c|}
\hline Distribution of Renal calculi according to $\operatorname{sex}(\mathbf{n}=\mathbf{7 0})$ & Frequency (\%) \\
\hline Gender & $55(78.0)$ \\
\hline Male & $15(22.0)$ \\
\hline Female & $70(100.0)$ \\
\hline Total & \multicolumn{2}{|c|}{ Table-1: Distribution of Renal calculi according to sex $(\mathbf{n}=70)$} \\
\hline \multicolumn{2}{|r|}{} \\
\hline
\end{tabular}

\begin{tabular}{|l|c|l|l|}
\hline Kidney stone type & Population & Color & Sensitivity \\
\hline Calcium oxalate & $80 \%$ & Black/ dark brown & Radio-opaque \\
\hline Calcium Phosphate & $10-15 \%$ & Dirty white & Radio Opaque \\
\hline Uric acid & $10-15 \%$ & Yellow/Reddish brown & Radiolucent \\
\hline Struvite & $5-10 \%$ & Dirty white & Radio Opaque \\
\hline Cystine & $1-2 \%$ & Pink/yellow & Radio-opaque \\
\hline \multicolumn{2}{|l}{} \\
\hline
\end{tabular}

\begin{tabular}{|l|c|}
\hline \multicolumn{2}{|l|}{ Distribution of Renal calculi in the study population $(\mathbf{n}=\mathbf{7 0})$} \\
\hline Type of stone former & Frequency $(\%)$ \\
\hline Single & $20(28.0)$ \\
\hline Bilateral & $40(58.0)$ \\
\hline Multiple & $10(14.0)$ \\
\hline Table-3: Distribution of Renal calculi in the study population \\
$(\mathrm{n}=70)$
\end{tabular}

body mass index (BMI), dietary pattern, family history, past history, average water intake, alcohol intake, type of stone, diagnostic tests, $24 \mathrm{hrs}$ urine analysis components, serum calcium, serum uric acid, serum creatinine, random blood sugar (RBS), and BUN and also other treatment methods used.

\section{STATISTICAL ANALYSIS}

Statistical analysis was done with the help of SPSS software. Chi square test was used for the comparison.

\section{RESULTS}

Total 70 patients diagnosed with either single, bilateral, or multiple, renal stones to the General Surgery Department of MNRMCH were included in this prospective study, Out of these 70 patients, 55 were males and 15 were Females diagnosed with renal calculi. It was observed that the frequency of stone formation was very high in males (78\%) when compared to females (22\%) (Table 1). The included variables were considered to be significant factors for the formation of stones if $\mathrm{p}<0.05$.

From 70 kidney stones analyzed (80\%) were Calcium oxalate $(\mathrm{CaOx})$ clearly predominated, followed by calcium phosphate (10-15\%), uric acid. (10-15\%) struvite (5-10\%) cysteine (1-2\%) described in Table 2.

About 28\% patients presented with single/unilateralstones, $58 \%$ bilateral stones and $14 \%$ with multiple stones (Table 3).

\section{DISCUSSION}

Nephrolithiasis has become a relatively common disorder, and its prevalence is greatly increasing worldwide. Any metabolic abnormality in an individual can result in single, bilateral or multiple stone. If this is left untreated, it can lead to recurrence of stones. The common metabolic abnormalities occurring in renal calculi patients are hypercalciuria, hyperuricosuria, hyperoxaluria, and hypocitraturia. Thus, evaluation of the metabolic abnormality and treating the patients can mostly reduce the incidence of stones. Metabolic evaluation(ME), is helpful for diagnosis and the risk of further recurrences in the future. This fact is supported by the study conducted by Joshi et al. ${ }^{6}$

In this study, $10-20 \%$ patients were obese, with increased body mass and can be another causative factor for renal stones. According to Taylor et al, it was concluded that obesity/weight gain can increase the risk of renal stone formation and that the magnitude of increased risk may be greater in women than in men. Weight gain in adulthood was associated with an increased risk of incident kidney stone formation in both men and women. Another study conducted by Hoppe (2003)et al. ${ }^{7}$ also revealed that higher BMI may be a risk factor for nephrolithiasis.

Shekarriz and Marshall ${ }^{8}$ according to this study laboratory evaluation should include urine analysis and culture;

Treatment of renal stones includes Medical expulsive therapy is used for spontaneous stone passage and In cases where medical expulsive therapy fails to pass the stone, other treatment opinions such as URS, ESWL, percutaneous nephrolithotomy (PCNL), and surgery are taken into consideration.

$\mathrm{Xu}$ et $\mathrm{al.}{ }^{9}$, in which he explained that low urine volume, abnormally urine $\mathrm{pH}$ and hyperuricosuria, leads to formation of uric acid stone. In such condition, the plan of treatment is to increase the solubility of uric acid in urine and to reduce its concentration by urinary alkalization using sodium or potassium alkali. The consumption of animal protein should also be greatly reduced. Goldfarb and Arowojolu ${ }^{10}$, it was said that predominant past, medical history mainly (diabetes and hypertension), they may be a contributor to stone formation.

\section{CONCLUSION}

Any individual with metabolic abnormality, there is chance to get stones in one or both the kidneys. It was observed that renal stone disease, was more common in the age group of $30-60$ and the mean age was observed to be $45.71 \pm 14.20$. It occurs in both gender, but the incidence was high in males than in females. The metabolic abnormalities were 
corrected by administration of suitable medications. Patients should be taught about the role of diet and water intake in the prevention of metabolic abnormalities and thereby prevention of renal stones.

\section{REFERENCES}

1. Reilly, RF, Jr, Perazella. Nephrolithiasis. p. 192-207.

2. Knoll,Thomas; Pearle,Margaret S.Clinical Management of Urolithiasis. Springer Science and Business Media. P. 21. ISBN 9783642287329

3. Preminger, GM, Cutler, RE, Merck Sharp and Dohme Corporation. "Stones in the Urinary Tract". The Merck Manual of Medical Information Home Edition. Whitehouse Station, New Jersey: Calium oxalates common In Committee to Review Dietary Reference Intakes for Vitamin D and Calcium 2011.

4. Gettman, MT, Segura, JW. Management of ureteric stones: Issues and controversies. British Journal of Urology International. 85-93.

5. Paterson RF. Arguments for a comprehensive metabolic evaluation of the first-time stone former. Can Urol Assoc J 2010;4(3):209-10.

6. Joshi A, Gupta SK, Srivastava A, Saudi J Kidney Dis Transpl. Metabolic evaluation in first-time renal stone formers in North India: A single center study. 2013;24(4):838-43.

7. Hoppe, B. Langman. A United States survey on diagnosis, treatment, and outcome of primary hyperoxaluria. Pediatric Nephrology. 18 (10): 986-91.

8. Shekarriz B, Marshall LS, Braz J Urol. Metabolic evaluation of stone disease. 2001;27(1):10-8.

9. $\mathrm{Xu} \mathrm{H}$, Zisman AL, Coe FL, Worcester EM. Kidney stones: An update on current pharmacological management and future directions. Expert Opin Pharmacother 2013;14(4):435-47.

10. Goldfarb DS, Arowojolu O. Metabolic evaluation of first-time and recurrent stone formers. Urol Clin North Am 2013;40(1):13-20.

Source of Support: Nil; Conflict of Interest: None

Submitted: 03-08-2018; Accepted: 20-08-2018; Published online: 19-09-2018 